\title{
A78 MECHANISMS AND CLINICAL RELEVANCE OF TRAIL- TRIGGERED RESPONSES IN SYNOVIAL FIBROBLASTS OF RHEUMATOID ARTHRITIS PATIENTS
}

Rachel Audo, ${ }^{1}$ Flavia Calmon-Hamaty, ${ }^{1}$ Dominique Baeten, ${ }^{4}$ Angelique Bruyer, ${ }^{1}$ Bernard Combe, ${ }^{1,3}$ Michael Hahne, Jacques Morel ${ }^{1,3}{ }^{1}$ Institut de Génétique Moléculaire de Montpellier, CNRS, UMR5535, Montpellier, France; ${ }^{2}$ Academic Medical Center, University of Amsterdam, Amsterdam, The Netherlands; ${ }^{3}$ Service d'Immuno-rhumatologie, CHU Lapeyronnie, Montpellier, France, ${ }^{4} \mathrm{MH}$ and JM share senior authorship

10.1136/ard.2010.149104.22

Objective We have shown that TRAIL induces apoptosis only in a portion of RA fibroblast-like synoviocytes (RAFLS) and that in the surviving cells, TRAIL induced proliferation. In the present study, we compared RAFLS-resistant and RAFLSsensible to TRAIL-induced apoptosis including levels of the TRAIL receptors (TRAIL-R) and clinical features of respective patient. We evaluated TRAIL and its soluble decoy receptor osteoprotegerin (OPG) levels in RA patients, osteoarthritis $(\mathrm{OA})$, spondylarthritis $(\mathrm{SpA})$.

Methods FLS were extracted from synovial tissues of RA patients $(n=30)$ and analysed by FACS for TRAIL-receptors expression. We obtained DAS28 within the 3 months of surgery for 13 patients. TRAIL-responses of FLS were analysed by AnnexinV for apoptosis, thymidine-incorporation for proliferation. TRAIL receptor activity was assessed by RNA silencing. HIC were performed to evaluate TRAIL level in synovial tissues from RA ( $n=7)$ and OA patients $(n=4)$. ELISA was used to determine TRAIL-levels in synovial fluid of OA; $n=20$ ), SpA; $(n=20)$ and establish RA patients $(n=30)$. Serum levels of TRAIL and OPG were measured in 72 patients fulfilling the ACR criteria (1987) with recent ( $<2$ years) and active ( $>3$ swollen joints) RA that were not treated or had a stable background treatment for at least 1 month. 48 of the RA patients were followed up at 6 months.

Results Disease severity of RA patients inversely correlated with susceptibility of FLS to TRAIL-induced apoptosis $(r=0.753, p=0.011)$. TRAIL-sensitive cells expressed significantly lower levels of the decoy TRAIL-R4 ( $p=0.008)$ and surprisingly also of TRAIL-R1 ( $p=0.014)$, one of the described death receptor. Silencing of these two receptors increased TRAIL-induced apoptosis in RAFLS. TRAIL levels were elevated in the arthritic joints of patients with established RA compared to other patients $(p<0.001)$. A low ratio OPG/TRAIL in sera of early RA patients at baseline was associated with a better evolution of disease activity $(p=0.028)$, but high serum levels of TRAIL at follow-up were associated with joint damages $(p=0.0063)$.

Conclusion Surprisingly, TRAIL-R1 seems to be a survival factor protecting RAFLS against TRAIL-induced apoptosis. 


\section{EWRR abstracts}

The negative correlation between TRAIL sensitivity in vitro and RA activity suggests that RAFLS develop resistance to escape TRAIL protective role. Indeed, in early RA patients, a low OPG/TRAIL ratio at baseline was associated with remission at 6 months but persistent TRAIL serum levels are associated with joint damage. These findings suggest a dual role for TRAIL in RA and resistance of RAFLS to TRAIL-induced apoptosis is associated with a disease promoting activity of TRAIL in RA. 